\title{
Design of the Distributed Control System Based on CAN Bus
}

\author{
Liang Chen \& Yingqing Guo \\ Northwestern Polytechnical University, Xi'an 710072, China \\ Tel: 86-29-8849-2310Ｅ-mail: chenliang@mail.nwpu.edu.cn
}

Received: June 5, 2011

Accepted: June 27, 2011

doi: $10.5539 /$ cis.v4n4p83

\begin{abstract}
The development trend of the aero-engine control system is the distributed control system (DCS), and the communication among devices is the key point and difficult point of DCS. In this article, the communication interface of CAN (Controller Area Network) bus is designed, the CAN communication technology among nodes is studied, the semi-physical platform of DCS is established, and the semi-physical simulation is implemented by combining with the engine model, and the experiment result shows that the control system simulation platform can completely satisfy the requirements of aero-engine DCS.
\end{abstract}

Keywords: Distributed control, CAN bus, Aero-engine

\section{Introduction}

CAN is a kind of multi-host area network which was proposed by BOSCH Company for the application of modern motor, and because of its high performance, high reliability, and real-time character, it has been widely applied in many departments such as industrial automation, multi-control equipment, vehicle, medical instruments, building, and environment control.

At present, the research about the DCS of aero-engine of China is still in the start stage, and the communication of the data bus of distributed system has not been solved essentially, so it is very difficult to construct the DCS of aero-engine, but the CAN bus can provide good solution for the design of the distributed system.

In the design of the aero-engine DCS, the semi-physical simulation platform of engine DCS is constructed on the system architecture, and the communication among various functional nodes is realized by the CAN bus, combining with requirements of the DCS simulation of aero-engine, and the semi-physical simulation of the engine model is realized finally, and the result of the communication experiment shows that the designed communication scheme can make the data to be transmitted with high speed and accuracy among nodes through the CAN bus, which can achieve the control requirements of the control system.

\section{Overall design of aero-engine DCS}

The aero-engine distributed control simulation system based on CAN bus designed in this article is seen in Figure 1.

The aero-engine distributed control system is composed b FADEC and various intelligent devices. In this system, the communication among various intelligent devices, FADEC, and model computers is realized by the CAN bus. In this article, the development board ICTECK-F2812 based on TMS320F2812 chip is adopted to simulate various functional devices, and two industrial control computers are used to simulate the engine model and FADEC, and the communication among devices is realized by the CAN bus.

\section{Hardware design of various nodes}

\subsection{FADEC}

FADEC node is composed by the CAN bus adapter card PCL-841 and the industrial control computer made by the Advantech Company, and its hardware principle chart is seen in Figure 2. The software flow of FADEC control program is seen in Figure 3.

FADEC is the core component of the whole control system, and its functions concludes the setting of control parameters, the data receiving and transmitting, the running of control algorithm, controlled variables displayed by the dynamic curve, and fault alarm. In the running, FADEC displays the controlled variables of engine by the form of dynamic curves, and displays the dynamic performance parameters of the control when the system is steady. 


\subsection{Intelligent executive institution}

The intelligent executive institution adopts the scheme of "DSP chip TMS320F2812 chip of the integrated CAN controller + CAN receiver 82C250", and the hardware principle of the intelligent executive institution is seen in Figure 4.

After the intelligent executive institution receives the control order of the controller FADEC through the CAN bus, the DSP micro-controller operates the control program and produces the digital control signals to the model computer.

\subsection{Intelligent sensor}

Like the intelligent executive institution, the intelligent sensor also adopts the scheme of "DSP chip TMS320F2812 chip of the integrated CAN controller + CAN receiver 82C250", and the principle of the intelligent sensor is seen in Figure 5.

\section{Software design of CAN bus communication}

In the aero-engine control simulation system, the aero-engine real-time mathematic model runs in the model computer, and the simulation system requires that the control cycle should be completed in each $20 \mathrm{~ms}$. A completed control flow is seen in Figure 6. Introduce the timer in FADEC, and transit the control order to the bus in each $20 \mathrm{~ms}$. Two intelligent executive institutions respectively receive the data and transmit the processed data to the control model computer through the CAN bus, and the model computer processes the data according to the received order, and exports the controlled variables, and the exported controlled variables are received and processed by two intelligent sensors, and transmitted to FADEC through the intelligent sensors, and so one transmitter control cycle is completed. Various nodes are set as the CAN bus data receiving interrupt mode.

\subsection{Setting of the CAN communication protocol in the DCS key technologies}

The criterion of CAN2.0B only defines the physical layer and the data link layer, and in the practice the application layer should be developed also.

The CAN bus adopts the short-frame structure, and the longest length of the data section is 8 bytes, with short transmission time, and low interfered probability. In the aero-engine control domain, 8 bytes can satisfy common requirements for the control order, the work state, and the data testing. And at the same time, the data length of 8 bytes will not occupy too long bus time, and the real-time character of the control system can be guaranteed.

The key of the CAN communication protocol is the setting of the ID, and the ID of the CAN node includes not only the address of the node, but the priority of the communication, and the ID is smaller, the priority is higher, and the ID is seen in Figure 7.

Taking the establishment of the aero-engine DCS as the example, the table setting CAN ID can be designed according to many aspects such as the data precision, the coding and decoding speed, and the data storage character (seen in Table 1).

The DCS structure designed in this article includes that two transmission channels correspond with one controller (EEC) and the model computer, and the start and close order controlling the EEC are transmitted to the intelligent executive institution by the broadcast form, and other data transmission and the cutting order are communicated by the form of point to point.

According to above coding rules, the data transmission order format transmitting to the executive institution of channel 1 is $0-01-01-10$, and data transmission order format transmitting to the executive institution of channel 2 is $0-10-11-10$. The broadcast order format is $1-\mathrm{xx}-01-0 \mathrm{x}$ where " $\mathrm{x}$ " represents 0 or 1 .

\subsection{Control algorithm machine and the computer implementation of mathematical model}

\subsubsection{Engine model program}

By the system identification method, the mathematical model of certain one single-variable small fanjet in the state with the speed of $89.9 \% \sim-94 \%$ can be denoted as follows. The input quantity (control quantity) is the fuel quantity WF, and the output quantity (controlled quantity) is the rotor speed $\mathrm{n}$.

$$
G(s)=\frac{n(s)}{w f(s)}=\frac{35.91 s+98.9}{s^{2}+4.097 s+14.83}
$$

Through the linear transformation, its discrete model can be denoted as follows. 


$$
G 1(z)=\frac{N(K)}{W F(K)}=\frac{0.354 z^{2}+0.01897 z-0.335}{z^{2}-1.916 z+0.9214}
$$

So,

$$
\begin{aligned}
N(K)= & 0.354 W F(K)+0.01897 W F(K-1)-0.335 W F(K-2) \\
& +1.916 N(K-1)-0.9214 N(K-2)
\end{aligned}
$$

The computer program of $\mathrm{C}$ language is

"N[2] = 0.354*WF[2]+0.01897*WF[1] $-0.335 * \mathrm{WF}[0]+1.916 \mathrm{~N}[1]-0.9214 \mathrm{~N}[0]$;

$\mathrm{N}[0]=\mathrm{N}[1]$;

$\mathrm{N}[1]=\mathrm{N}[2]$

$\mathrm{WF}[0]=\mathrm{WF}[1]$

$\mathrm{WF}[1]=\mathrm{WF}[2] ;$

4.2.2 Central control algorithm based on PID

The digital PID controller is

$\frac{U(z)}{E(z)}=K p+\frac{K i T(z+1)}{2(z-1)}+\frac{K d(z-1)}{T z}$

In above formula, $\mathrm{T}$ is the sampling period of the system, $0.02 \mathrm{~s}$. From the above formula,

$U(k)=$

$\frac{(100 K p+K i+5000 K d)}{100} E(k)+\frac{(K i-100 K p-10000 K d)}{100} E(k-1)+50 K d E(k-2)+U(k-1)$

In the designed PID controller, $\mathrm{Kp}=0.51, \mathrm{Ki}=1.52$, and $\mathrm{Kd}=0.01$.

\section{Test of experiment result}

By implementing the semi-physical simulation of the engine model on the distributed system platform, the rotate speed curves of two channels are seen in Figure 8, and the dynamic character parameter of the control system is seen in Figure 9, and the experiment result shows that the system has quick dynamic response speed and small overshoot, which can achieve the control requirements.

The distributed control system designed in this article chooses two intelligent sensors and two intelligent executive institution nodes, and the controlled object is the engine model with single variable, so in the simulation experiment, the EEC node will export the control quantities to the model computer through two control loops in the similar work states, and then the control quantities are collected by the intelligent sensors in their own loops through the operation of the engine model program and transmitted to the EEC node, and finally the PID control algorithm are respectively performed. From the figure, the rotate speed change curves collected by two channels are almost same. The simulation experiment proves the exactness and generality of the aero-engine DCS principle prototype established in this article.

\section{Conclusions}

The aero-engine distributed control system designed is designed in this article, and the experiment result shows that the data can be transmitted correctly with high speed and certain cycle among various nodes through the CAN bus, and the special advantage of the CAN bus in the design of the distributed control system can satisfy the requirements to construct the distributed control system.

\section{References}

Dr. Ramesh Rajagopalan, Bruce C. Wood, Matthew Schryver. (2003). Evolution of Propulsion Controls and Health Monitoring at Pratt and Whitney. AIAA. No. 2645.

Guo, Yingqing. (2003). Survey of the Distributed Control System for an Aero-engine. Aeroengine. No. 29(3).

H.A. Thompson, H. Benitez-Perez, D. Lee, D.N. Ramos-Hernandez, P.J. Fleming, C.G. Legge. (1999). A CAN bus-based safety-critical distributed aero-engine control systems architecture demonstrator. Microprocessors and 
Microsystems. No. 23. P. 345-355.

Huang, Jinquan \& Xu, Ke. (2003). Distributed Control Systems for Aeroengines: A Survey. Journal of Aerospace Power. Vol. 18. No.5. P. 698-704.

Li, Guangyao. (2009). Communication Research on CAN Based on Distributed Aero-engine Control System. Measurement \& Control Technology. No.1.

P. L. Shaffer. (1999). Distributed Control System for Turbine Engines. ASME. 102/Vol. 121. January, 1999

TMS320F28x. (2002). Enhanced Controller Area Network (eCAN) Peripheral Reference Guide. Texas Instruments. May of 2002.

Table 1. Order distribution

\begin{tabular}{|c|c|c|c|c|c|}
\hline \multirow[b]{2}{*}{ Control device } & \multirow{2}{*}{$\begin{array}{l}\text { Receiving ID Number } \\
\text { (ID24-18) }\end{array}$} & \multicolumn{3}{|c|}{ Control order } & \multirow{2}{*}{\begin{tabular}{|c|} 
Control data \\
Data \\
transmission
\end{tabular}} \\
\hline & & Start & Shut & Cut off & \\
\hline Controller & $\mathrm{xxx} 00 \mathrm{xx}$ & $1 \times x 0100$ & $1 \times x 0101$ & $0 \mathrm{xx} 0111$ & $0 \times x 0110$ \\
\hline Executive institution & $\mathrm{xxx} 01 \mathrm{xx}$ & $0 \times x 1000$ & $0 \times x 1001$ & $0 \times x 1011$ & $0 \times x 1010$ \\
\hline Engine & $\operatorname{xxx} 10 x x$ & $0 \times x 1100$ & $0 \times x 1101$ & $0 \times x 1111$ & $0 \times x 1110$ \\
\hline Sensor & $\mathrm{xxx} 11 \mathrm{xx}$ & $0 \times x 0000$ & $0 \times x 0001$ & $0 \times x 0011$ & $0 \times x 0010$ \\
\hline ID24 & ID22, 23 & \multicolumn{2}{|c|}{ ID20, 21} & \multicolumn{2}{|c|}{\begin{tabular}{l|l} 
& ID18, 19
\end{tabular}} \\
\hline Broadcast order sign & Channel sign & \multicolumn{2}{|c|}{ Executive component sign } & \multicolumn{2}{|c|}{ Control order } \\
\hline
\end{tabular}

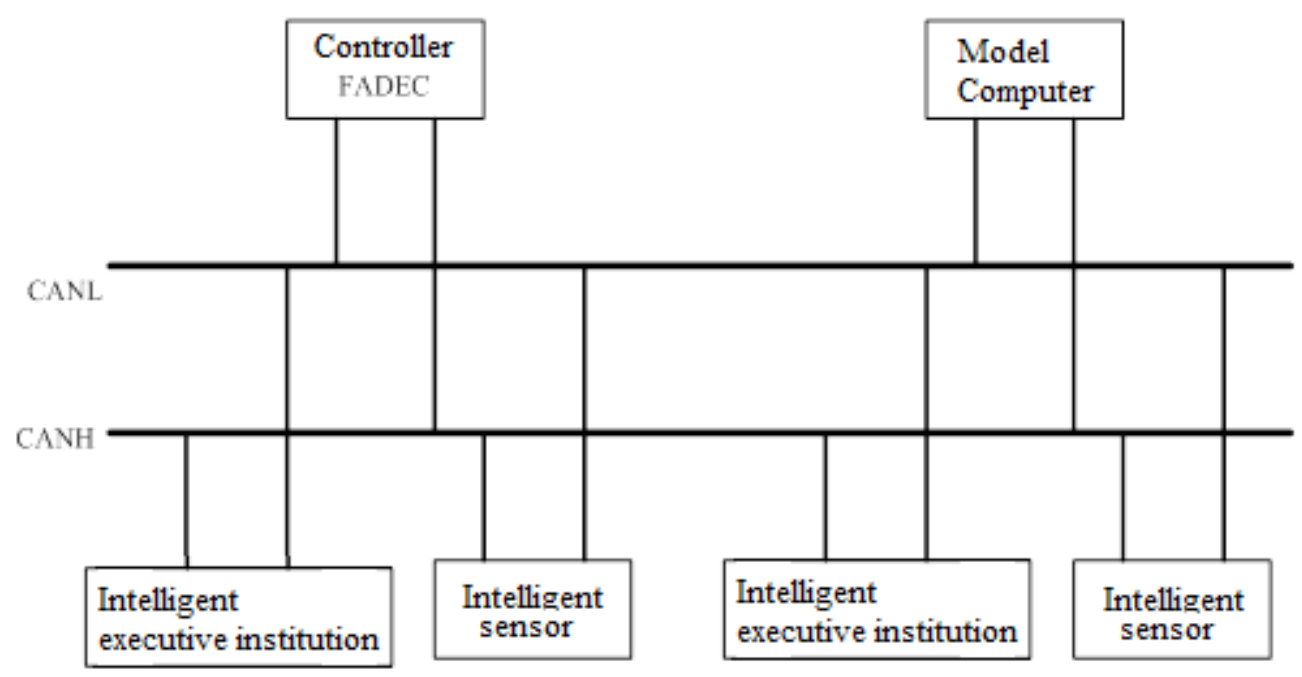

Figure 1. Aero-engine Distributed Control Simulation System Based on CAN Bus 


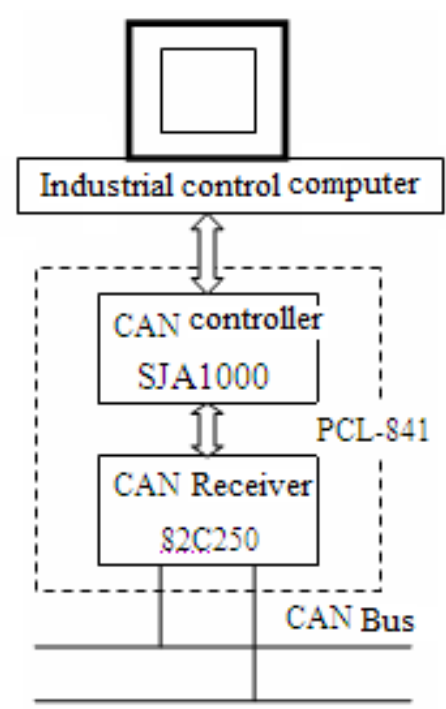

Figure 2. Hardware Principle of FADEC

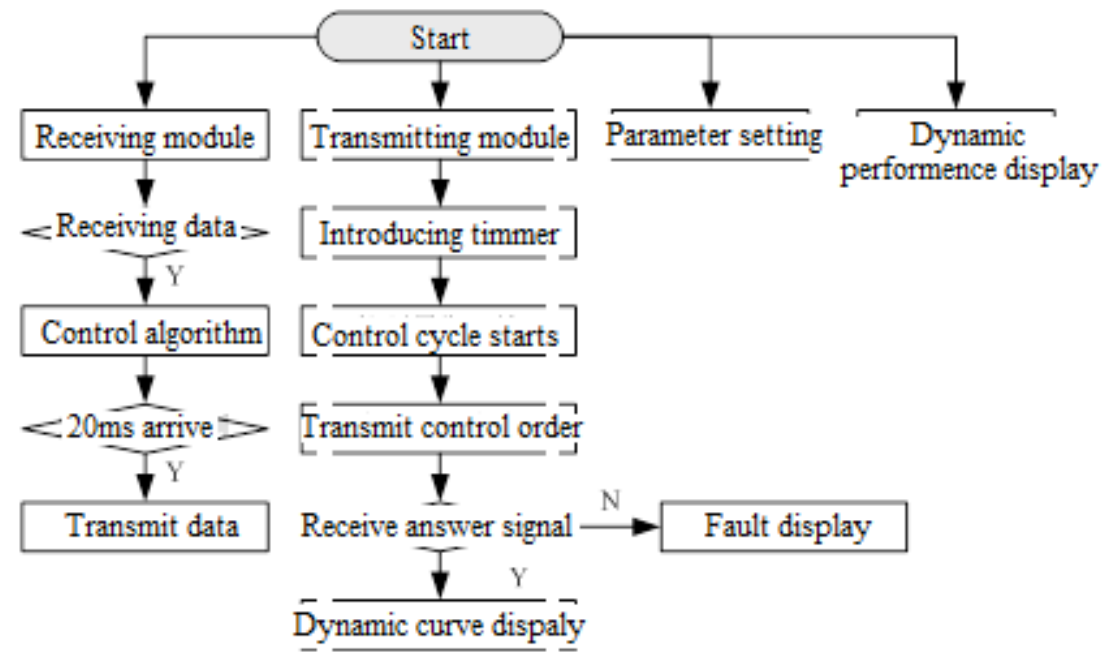

Figure 3. Software Flow of FADEC Node

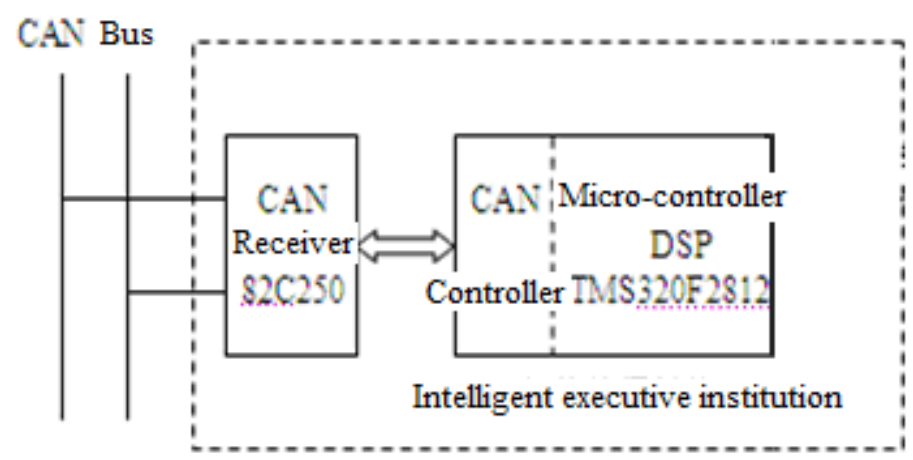

Figure 4. Hardware Software of Intelligent Executive Institution 


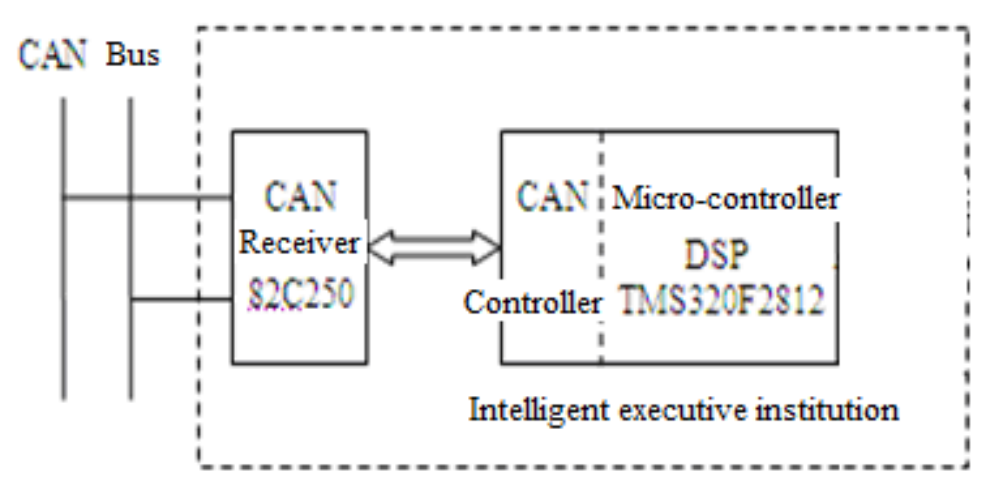

Figure 5. Hardware Principle of Intelligent Sensor

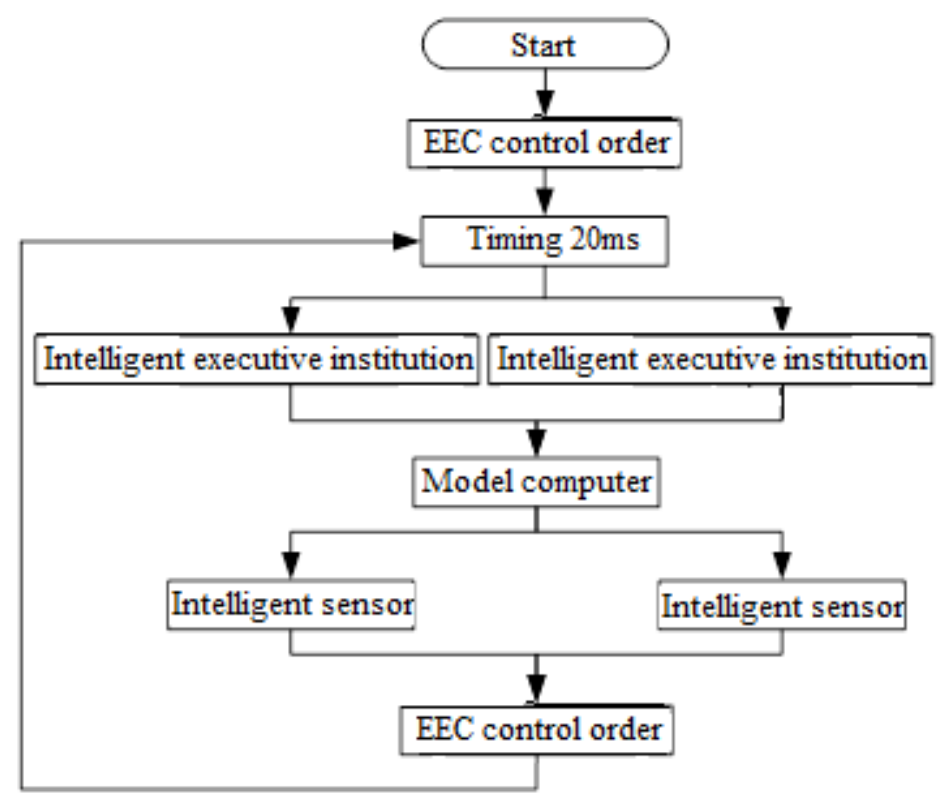

Figure 6. Control Flow Chart

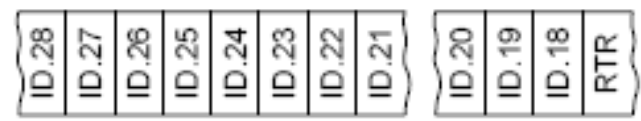

Figure 7. Distribution of CAN ID

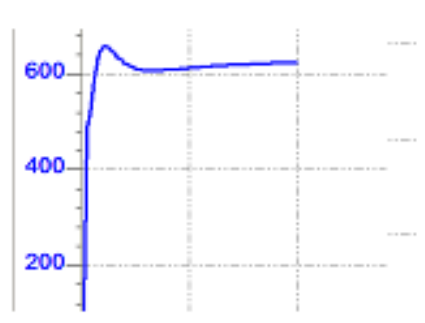

Channel 1

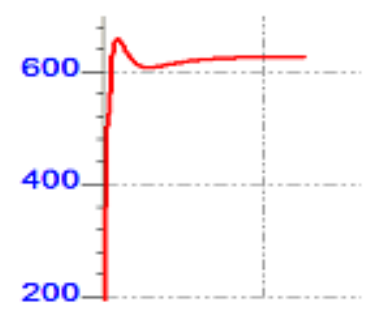

Channel 2

Figure 8. Simulation Curve of the DCS Hardware in the Loop Aiming at Certain One Single-Variable Engine 


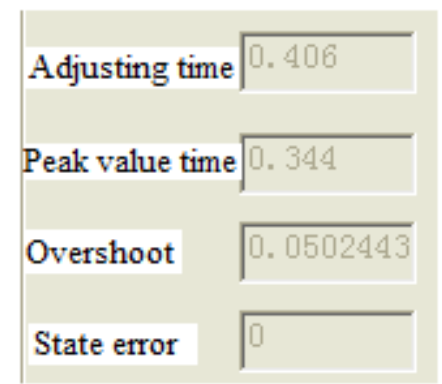

Figure 9. Dynamic Performance of Control System 